\title{
Calculation of Field Quality in Fast-Ramping Superconducting Magnets
}

\author{
Bernhard Auchmann, Riccardo deMaria, and Stephan Russenschuck
}

\begin{abstract}
Fast-ramping superconducting (SC) accelerator magnets are the subject of $R \& D$ efforts at various laboratories. The simulation of field quality in fast-ramping magnets requires modifications of magnet design tools such as the CERN field computation program ROXIE. In this paper we present the efforts towards dynamic 2-D simulations of fast-ramping SC magnets. Models for persistent currents, inter-strand coupling currents, inter-filament coupling currents, and for eddy-currents in conducting coil-wedges are described and validated.
\end{abstract}

Index Terms-Field quality, transient effects.

\section{INTRODUCTION}

$\mathbf{T}$ HE ROXIE program is developed for the design of superconducting (SC) magnets [1]. The coil fields and potentials are calculated from Biot-Savart type integrals and the yoke magnetization is determined using a coupling method of finite elements and boundary elements. In addition, a model for persistent currents (PCs) based on the critical state model is implemented for the quasi-static simulation of SC magnets.

For the simulation of fast-ramping magnets, additional eddycurrent related effects need to be modeled. Eddy currents in the laminated yoke, which occur mainly in the 3-D end region of a magnet, are not the subject of this paper. We present models for inter-filament coupling currents (IFCCs) and for inter-strand coupling currents (ISCCs) as well as for eddy currents induced in the coil's copper wedges. We emphasize the necessity to calculate the mutual interdependence of these effects, while considering the non-linear effects from iron magnetization.

For the FAIR-project (Facility for Antiproton and Ion Research) at the "Gesellschaft für Schwerionenforschung" (GSI), a model dipole called GSI001 was built at Brookhaven National Laboratory (BNL), with a nominal field of $4 \mathrm{~T}$ and ramp rates of up to $4 \mathrm{~T} / \mathrm{s}$. The magnet design is similar to an existing dipole at BNL, with some changes for loss reduction and improved cooling. The magnet length is approximately $1.2 \mathrm{~m}$. Measurements of field quality and losses have been carried out at BNL at different ramp rates [2]. We use this magnet for simulations and software validation.

Finally, we discuss the impact of eddy currents in conducting coil-wedges on the field quality, in the context of an upgrade study for the SPS proton synchrotron at CERN [3]. The 2-D numerical modeling is not trivial. We sketch the mathematical model and give first simulation results.

Manuscript received August 28, 2007.

B. Auchmann and S. Russenschuck are with CERN AT/MEL, CH-1211 Geneva 23, Switzerland (e-mail: bernhard.auchmann@cern.ch).

$\mathrm{R}$. de Maria is with CERN AB/ABP, CH-1211 Geneva 23, Switzerland.

Color versions of one or more of the figures in this paper are available online at http://ieeexplore.ieee.org.

Digital Object Identifier 10.1109/TASC.2008.922247

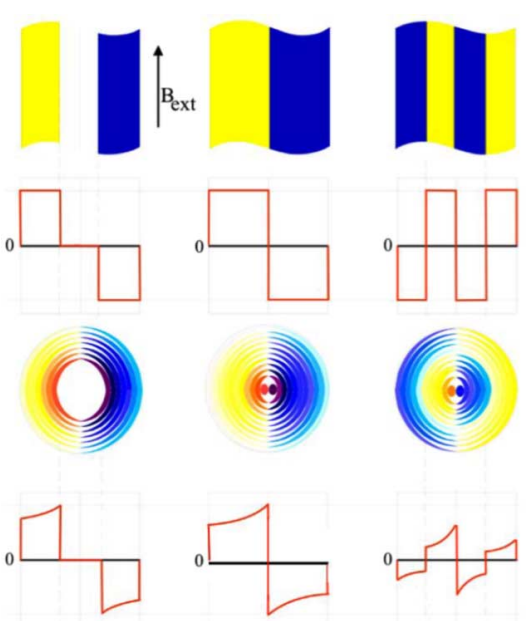

Fig. 1. Top: Shielding currents according to the critical state model in a SC slab with parallel external field. Bottom: Shielding currents according to the refined critical state model in round filaments. In the left plots the external field is increased from zero to an external induction $B_{\text {ext }}$. In the middle plot, the field is further increased until it fully penetrates the SC material. In the right plot the field is decreased again, inducing a new layer of shielding currents.

\section{EDDY-CURRENT EFFECTS IN SC COILS}

In this section we present the mathematical models for persistent currents, inter-filament coupling currents, and inter-strand coupling currents.

\section{A. Persistent Currents}

According to the critical state model by Bean [4] the (persistent) currents that shield the inside of a Type-II superconducting slab always flow at the critical current density $J_{\mathrm{c}}$ of the material, Fig. 1 (top). Wilson [5] applied the critical state model to a circular cylinder in a transverse field to describe persistent currents in filaments. Aleksa et al. [6] refined the Wilson model using intersecting circles and ellipses, in order to account for the inhomogeneous critical current density inside one layer of shielding currents. The inhomogeneity is due to the fact that $J_{\mathrm{c}}$ depends on the local magnetic induction Fig. 1 (bottom). This method allows to explain the so-called peak-shifting effect in the M(B) curve and allows for the calculation of minor hysteresis loops.

The magnetization of one layer of shielding currents between normalized radii $q_{i}$ and $q_{i+1}$ is calculated by

$$
\begin{aligned}
M_{\mathrm{PC}, i} & =\lambda_{\mathrm{f}} \frac{4 r}{\pi} \int_{q_{i}}^{q_{i+1}} J_{c}(B(q), T)(1-q)^{2} \mathrm{~d} q \\
& \approx \lambda_{\mathrm{f}} \frac{4 r \mathcal{F}}{\pi} \int_{q_{i}}^{q_{i+1}} \frac{(1-q)^{2}}{\sqrt{B(q)}} \mathrm{d} q,
\end{aligned}
$$




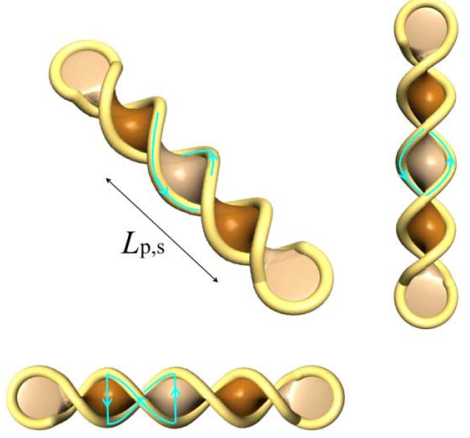

Fig. 2. Eddy-current loop between two twisted filaments in a resistive matrix. Visualization with SeifertView [7].

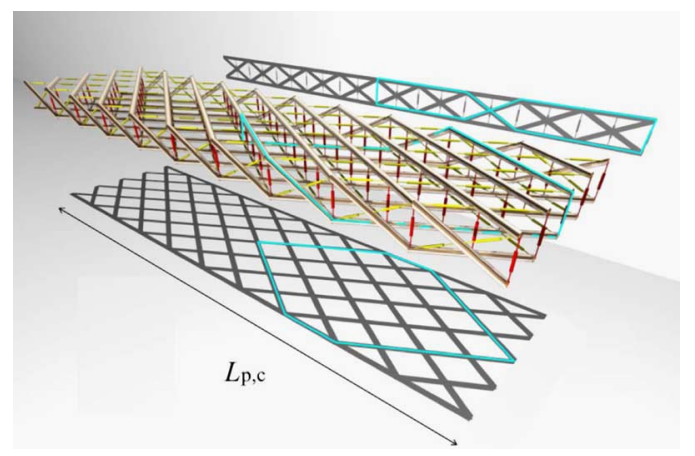

Fig. 3. Electrical network representing one twist-pitch length of a Rutherfordtype cable. Adjacent resistances between neighboring strands are depicted in yellow and cross-over resistances in red.

where $\mathcal{F}=J_{\mathrm{c}}\left(B_{\text {ext }}, T\right) \sqrt{B_{\text {ext }}}$ with $B_{\text {ext }}$ denoting the magnetic induction outside the filament, the filament radius $r$, and the filling factor $\lambda_{\mathrm{f}}$ of filaments in a strand [6].

\section{B. Inter-Filament Coupling Currents}

The filaments in a strand are twisted with a characteristic twist pitch $L_{\mathrm{p}, \mathrm{s}}$. The filaments are imbedded in a matrix with an effective resistivity $\rho_{\text {eff. }}$. Eddy currents are induced in loops of a length of up to half a twist pitch, bounded by the superconducting filaments and closed across the resistive matrix, Fig. 2. The resulting magnetization produced by eddy currents in these loops can be calculated as [5]

$$
M_{\mathrm{IF}}=\lambda_{\mathrm{s}} \partial_{t} B \frac{L_{\mathrm{p}, \mathrm{s}}}{2 \pi} \underbrace{\frac{1}{\rho_{0}+\rho_{1} B}}_{\rho_{\text {eff }}},
$$

where $\lambda_{\mathrm{s}}$ is the filling factor of twisted filaments in a strand, $\rho_{0}$ is the constant part of the effective resistivity and $\rho_{1}$ the slope of the magneto-resistive effect.

\section{Inter-Strand Coupling Currents}

On the scale of half a conductor twist-pitch of Rutherfordtype cable we find loops that are bounded by superconducting strand branches, and cross- and adjacent resistances. In order to consider all relevant loops we model the Rutherford cable as an electrical network [8], Fig. 3. The sources in the electrical network are the time derivatives of the integrated magnetic vector potential along the branches of the network. Let $[\mathrm{M}]$ denote the

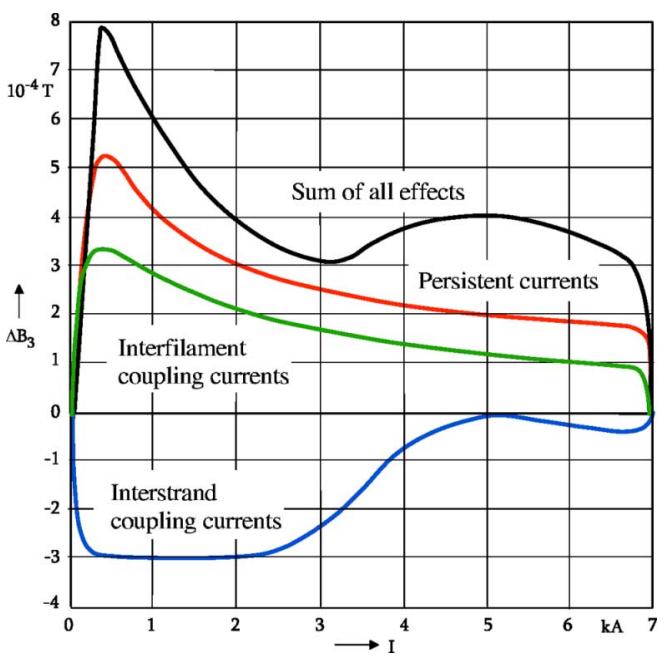

Fig. 4. Typical signatures in the $\Delta B_{3}$ graph of PCs, IFCCs, and ISCCs.

mesh matrix of the network and $[\mathrm{R}]$ the resistance matrix, $\{I\}$ the vector of the branch currents and $\left\{\partial_{t} A\right\}$ the induced voltages. We can solve for the currents by evaluating

$$
\{I\}=-[\mathrm{M}]\left([\mathrm{M}][\mathrm{R}][\mathrm{M}]^{\mathrm{T}}\right)^{-1}[\mathrm{M}]\left\{\partial_{t} A\right\} .
$$

\section{COMPARISON TO MEASUREMENTS}

The GSI001 magnet was built with a slightly adapted RHIC coil design. The cable contains a $25 \mu \mathrm{m}$ stainless-steel core to increase the cross-over resistance and thus to reduce ISCCs. The GSI001 dipole was powered between 0 and $4 \mathrm{~T}$ central field with ramp rates varying from 0 to $4 \mathrm{~T} / \mathrm{s}$.

To analyze the contribution of the time transient effects to the field distortions we compare the difference in the sextupole component (given in Tesla) between the up- and down-ramp. The multipole content is analyzed at a reference radius $r_{0}$ of $25 \mathrm{~mm}$. Measurement data of GSI001 was supplied in [9].

\section{A. Signatures in the $\Delta B_{3}$ Plot}

PCs and IFCCs have a similar signature in the $\Delta B_{3}$ plot that shows the difference in the sextupole component between upand down-ramp, see Fig. 4. Both effects are inversely proportional to the magnetic induction. For PCs this is due to the critical current density in superconductor, and for IFCCs the reason lies in the magneto-resistance of the resistive matrix. IFCCs depend linearly on the ramp rate. ISCCs have a different signature. Their contribution to the $\Delta B_{3}$ plot has opposite sign. Surprisingly, also the ISCC contribution reduces with the magnetic induction! We observe that the ISCCs fade away when iron saturation sets in and the flux distribution across the cables changes, i.e., the net flux through the broad side of the cables is reduced, Fig. 5.

\section{B. Simulation with Nominal Material Parameters}

We recall the nominal material parameters [10] of the above models and show the comparison of measurements and simulation results for the GSI001 magnet. 

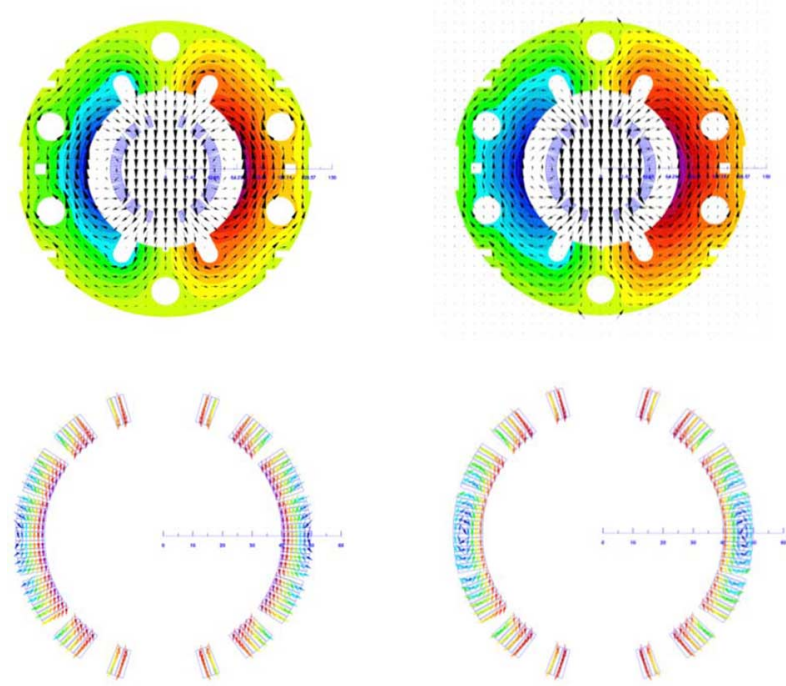

Fig. 5. Distribution of magnetic flux in the GSI001 magnet at low excitation (left) and high excitation (right). We observe that with increasing field, the vertex of the magnetic flux is displaced towards the center of the magnet.

$\Delta \mathrm{B} 3$ (tesla) Nominal Material Parameters

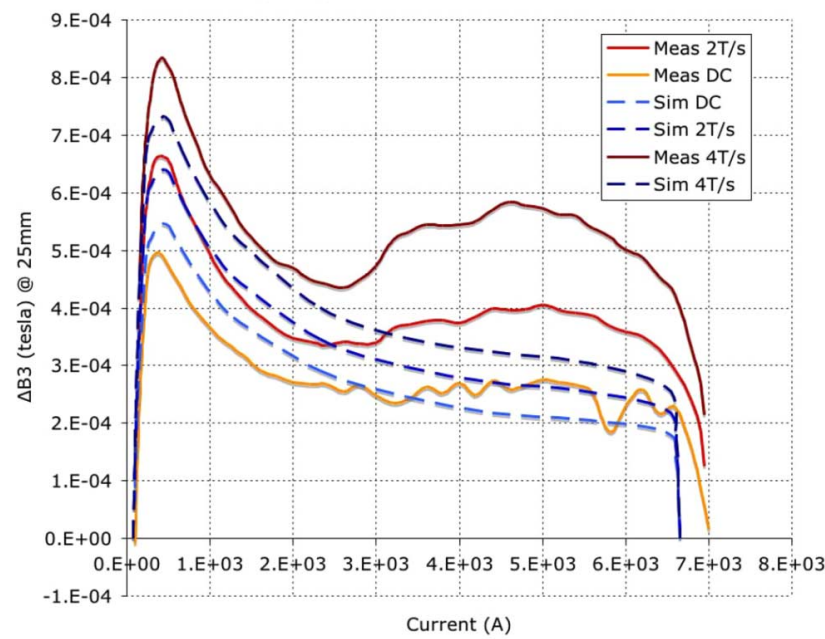

Fig. 6. Difference in the absolute sextupole (in tesla) between up- and down ramp between 0 and $4 \mathrm{~T}$ in the aperture of the GSI001 dipole at DC conditions, $2 \mathrm{~T} / \mathrm{s}$, and $4 \mathrm{~T} / \mathrm{s}$ ramp rates. Measurement results are shown in red, simulations in blue with dashed lines. Simulations use nominal material parameters.

The critical current density as a function of the temperature $T$ and the modulus of the magnetic induction $B=|\mathbf{B}|$ is given by the following fit [11], where $B_{\mathrm{c}}=B_{\mathrm{c} 20}\left(1-\left(T / T_{\mathrm{c} 0}\right)^{1.7}\right)$

$$
J_{\mathrm{c}}(B, T)=\frac{J_{c}^{\mathrm{ref}} C_{0} B^{\alpha-1}}{\left(B_{c}\right)^{\alpha}}\left(1-\frac{B}{B_{c}}\right)^{\beta}\left(1-\left(\frac{T}{T_{\mathrm{c} 0}}\right)^{1.7}\right)^{\gamma}
$$

with parameters for Nb-Ti of $J_{\mathrm{c}}^{\text {ref }}=3 \cdot 10^{9} \mathrm{Am}^{-2}, B_{\mathrm{c} 20}=$ $14.5 \mathrm{~T}, T_{\mathrm{c} 0}=9.2 \mathrm{~K}, C_{0}=27.04 \mathrm{~T}, \alpha=0.57, \beta=0.9$, and $\gamma=2.32$. The filament diameter is $6 \mu \mathrm{m}$ and the non-superconductor to superconductor ratio in the strand is 2.21 . The parameters for IFCCs are $\rho_{0}=1.24 \cdot 10^{-10} \Omega \mathrm{m}^{-1}, \rho_{1}=$ $9 \cdot 10^{-11} \Omega \mathrm{m}^{-1} \mathrm{~T}^{-1}$, and $\lambda_{\mathrm{S}}=0.5$, with a twist-pitch length $L_{\mathrm{p}, \mathrm{s}}=4 \mathrm{~mm}$. The ISCC model uses the following parameters: number of strands per conductor 30, twist-pitch length $74 \mathrm{~mm}$, $R_{\mathrm{a}}=6.4 \cdot 10^{-5} \Omega$, and $R_{\mathrm{c}}=6.25 \cdot 10^{-2} \Omega$. Results obtained with these parameters are shown in Fig. 6.

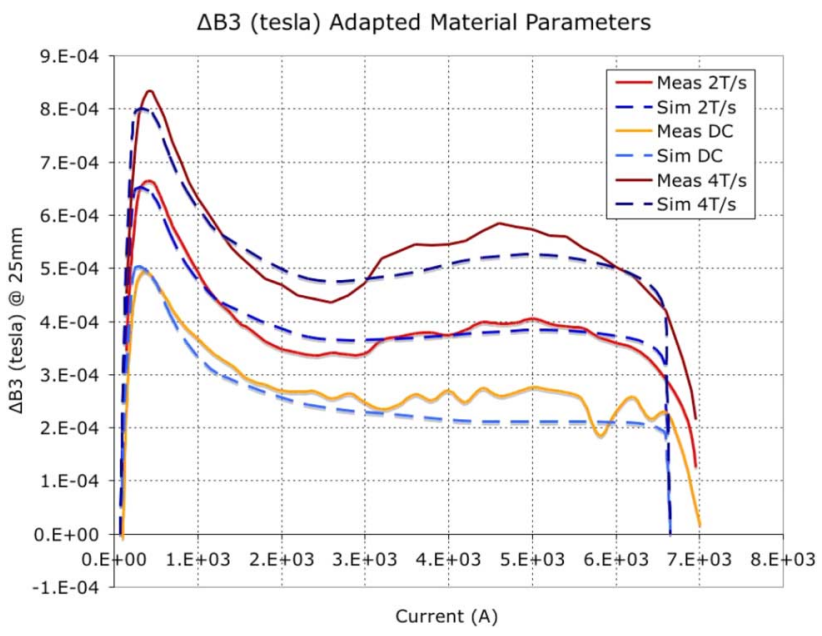

Fig. 7. Difference in the absolute sextupole (in tesla) between up- and downramp between 0 and $4 \mathrm{~T}$ in the aperture of the GSI001 dipole at DC conditions, $2 \mathrm{~T} / \mathrm{s}$, and $4 \mathrm{~T} / \mathrm{s}$ ramp rates. Measurement results are shown in red, simulations in blue with dashed lines. Simulations use adapted material parameters in order to better reproduce the measurements.

\section{Simulation with Adapted Material Parameters}

Some effort was put into the reconstruction of the measured $\Delta B_{3}$ curves at 0,2 , and $4 \mathrm{~T} / \mathrm{s}$. We present the parameters that reproduce the measurements reasonably well. For the DC curve a different critical current fit was required, as the flat curve in the high-field region could not be reproduced with the above function. The fit reads [12]

$$
\begin{array}{r}
J_{\mathrm{c}}(B, T)=J_{\mathrm{c}}^{\mathrm{ref}} C_{0}\left(C_{1} \mathrm{e}^{-B \alpha_{1}}+1\right)\left(C_{2} \mathrm{e}^{-B \alpha_{2}}+1\right) \\
\left(1-\frac{B}{B_{c}}\right)^{\beta}\left(1-\left(\frac{T}{T_{\mathrm{c} 0}}\right)^{1.7}\right)^{\gamma},
\end{array}
$$

with $B_{\mathrm{c}}$ defined above and with the parameters $J_{\mathrm{c}}^{\text {ref }}=3$. $10^{9} \mathrm{Am}^{-2}, B_{\mathrm{c} 20}=14.5 \mathrm{~T}, T_{\mathrm{c} 0}=9.2 \mathrm{~K}, C_{0}=6.5, C_{1}=$ $2, C_{2}=2, \alpha_{1}=6 \mathrm{~T}^{-1}, \alpha_{2}=15 \mathrm{~T}^{-1}, \beta=0.5$, and $\gamma=2.32$. For IFCCs we use $\rho_{0}=9 \cdot 10^{-11} \Omega \mathrm{m}^{-1}, \rho_{1}=$ $3 \cdot 10^{-11} \Omega \mathrm{m}^{-1} \mathrm{~T}^{-1}, \lambda_{\mathrm{S}}=0.7$. The ISCC resistances are set to $R_{\mathrm{a}}=1.8 \cdot 10^{-5} \Omega$, and $R_{\mathrm{c}}=6.25 \cdot 10^{-2} \Omega$. Results from these parameters are shown in Fig. 7. The measurements can thus be well explained, if a three to four times lower adjacent resistance (with respect to the nominal value) is taken into account.

\section{EDdy CURRENTS IN WEDGES}

We discuss field distortions and losses due to eddy currents in conducting coil-wedges. A standard 2-D FEM code is not suited for this kind of calculations. We sketch the adjusted mathematical model and give first simulation results. The calculations were carried out in the context of an upgrade study for the SPS proton synchrotron at CERN [3].

\section{A. Theory}

The fundamental assumption for applying 2-D finite elements (FEM) to eddy current simulations is an infinitely long geometry with all fields constant along the longitudinal axis. The fields are either directed longitudinally or transversally. It is common knowledge that this assumption implies a Maxwell-gauge of 


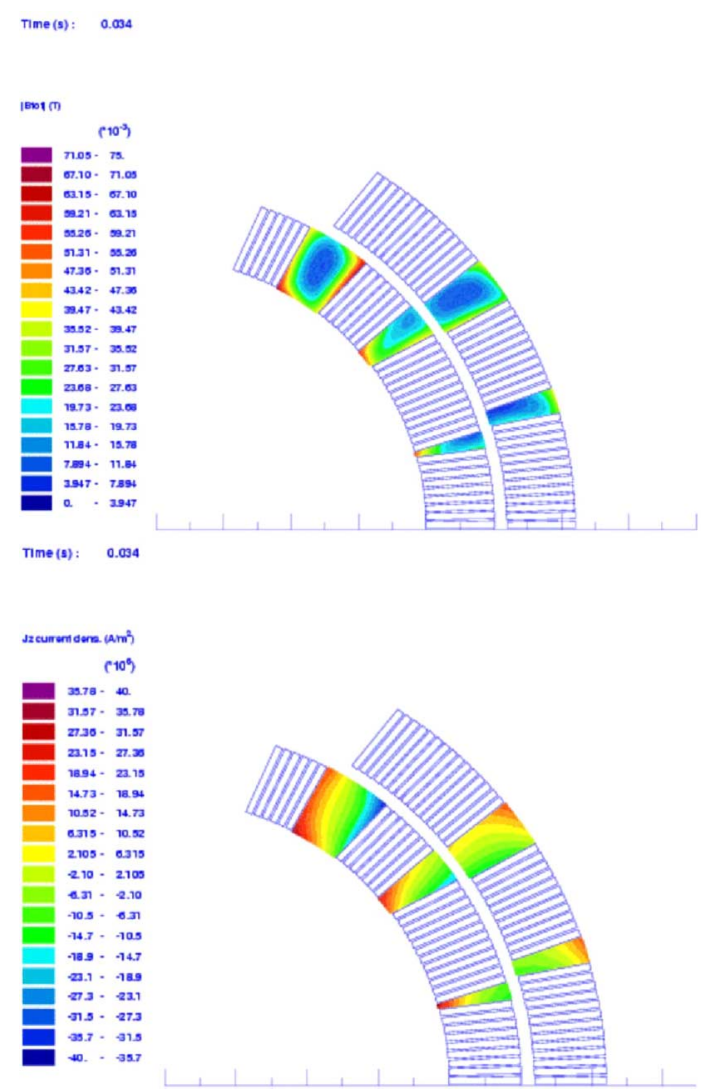

Fig. 8. Top: The magnetic induction is shielded from the inside of the wedges. Bottom: Eddy currents in the wedges.

the magnetic vector potential ( $\operatorname{div} \mathbf{A}=0$ ). It is rarely mentioned, however, that this assumption also implies that the electric scalar potential is constant over the entire domain of interest ( $\varphi=$ const.). Physically this represents the short-circuiting of all conductive elements at infinity. The magnetic flux between any two conductive elements is linked in a loop closed at infinity. Consequently, large eddy currents can flow from one conductive element to another. This is, however, not the case in SC magnets where the wedges are of finite length $(3.6 \mathrm{~m}$ in the LHC main dipoles) and are electrically insulated with respect to each other.

We thus need to introduce one additional degree of freedom per conductive element into the system of FEM equations, compare red coefficients in (6a). These degrees of freedom represent longitudinal electric voltages. Furthermore the additional equations ensure that the Faraday law is obeyed. The net eddy-current flow in each conductive element can now be specified, e.g., set to zero on the right-hand side of (6b).

$$
\begin{aligned}
\underbrace{[\mathrm{D}]^{1}\left[\mathrm{M}_{\nu}^{1}\right]\left[\mathrm{D}^{0}\right]\left\{A_{z}\right\}}_{\text {curl } \nu \text { curl } \mathbf{A}_{z}} & +\underbrace{\left[\mathrm{M}_{\kappa}^{1}\right]\left\{A_{z}\right\}}_{\kappa \partial_{t} \mathbf{A}_{z}} \\
& -\underbrace{\left[\mathrm{P}_{\mathrm{w}}^{2}\right][\tau]\left[\mathrm{G}^{\prime}\right]\left[\mathrm{U}^{\prime}\right]}_{\tau G^{\prime} U^{\prime}}=\left\{j_{\underline{s}}\right\} \\
& -\underbrace{[\tau]^{\mathrm{T}}\left[\mathrm{P}_{\mathrm{w}}^{2}\right]^{\mathrm{T}}\left\{\partial_{t} A_{z}\right\}}_{\partial_{t} \Psi_{1}}+\left\{U^{\prime}\right\}=\left[\mathrm{R}^{\prime}\right]\{I\}
\end{aligned}
$$

Equations (6a) and (6b) represent an adapted 2-D FEM vectorpotential formulation in the notation of discrete electromagnetism [13]. Matrices are in brackets, coefficient vectors in curly
TABLE I

JoUle Losses AND Field Distortions DUE TO EDDY CURRENTS IN WEDGES MADE OF DIFFERENT MATERIAL

\begin{tabular}{llll}
\hline Material $(4 \mathrm{~K})$ & Resistivity $(\Omega \cdot \mathrm{m})$ & Losses $(\mathrm{W} / \mathrm{m})$ & $\Delta b_{3}$ \\
\hline \hline pure copper & $1.7 \cdot 10^{-10}$ & 22.03 & 3.1 \\
bronze & $3.0 \cdot 10^{-8}$ & 0.13 & 0.01 \\
stainless steel & $5.0 \cdot 10^{-7}$ & $7.5 \cdot 10^{-3}$ & $5 \cdot 10^{-3}$ \\
\hline
\end{tabular}

braces. In (6a) we split the total current density curlH into a source current $\boldsymbol{j}_{\mathrm{S}}$, a conduction current $\tau G^{\prime} U^{\prime}$ and an induction current $\kappa \partial_{t} \mathbf{A}_{s}$. The $[\tau]$ matrix contains the inverse of wedge areas, $R^{\prime}$ is the resistance per unit length, $G^{\prime}=1 / R^{\prime}, \kappa$ is the conductivity and $\nu$ is the magnetic permeability. $\Psi_{1}$ represents the linked flux in a wedge, and $\left[\mathrm{P}_{\mathrm{w}}^{2}\right]$ is the pairing matrix.

\section{B. Results}

Eddy-current losses in the conductive wedges of a main dipole were calculated for an upgrade study of the SPS. To this end we couple the above adapted FEM formulation to the boundary-element method. The results are displayed Fig. 8. Numerical values are given in Table I for a ramp rate of $1.5 \mathrm{~T} / \mathrm{s}$. We find that only pure copper produces significant losses. The eddy-current density in the copper wedge is of the order of the transport current. The eddy currents therefore have a sizable impact on field quality.

\section{CONCLUSION}

We have validated the numerical models for the simulation of time transient effects in fast ramping superconducting magnets. It was shown that it is important to have all models available in one field-computation tool, in order to study the mutual interdependence of the effects. The models also serve in quench simulations for the calculation of losses.

\section{REFERENCES}

[1] S. Russenschuck, Electromagnetic Design and Mathematical Optimization Methods in Magnet Technology, 3rd ed. eBook at: http://cern.ch/russ, Feb. 2006.

[2] G. Moritz, J. Kaugerts, J. Escallier, G. Ganetis, A. K. Jain, A. Marone, J. F. Muratore, R. Thomas, P. Wanderer, B. Auchmann, S. Russenschuck, and R. de Maria, "Recent test results of the fast-pulsed $4 \mathrm{~T} \cos \theta$ dipole GSI001," in Proc. 2005 Particle Accelerator Conf., Knoxville, TN, Oct. 2005, pp. 683-686.

[3] G. Kirby, "Magnet design options for the LHC injection chain," in Proc. WAMDO 2006 Workshop at CERN, Mar. 2006, pp. 144-149.

[4] C. P. Bean, "Magnetization of hard superconductors," Phys. Rev. Lett., vol. 8, no. 6, pp. 250-253, Mar. 1962.

[5] M. N. Wilson, Superconducting Magnets. Oxford, U.K.: Oxford Science, 1983.

[6] M. Aleksa, C. Voellinger, and S. Russenschuck, "Magnetic field calculations including the impact of persistent currents in superconducting filaments," IEEE Trans. Magn., vol. 38, no. 2, pp. 825-828, Mar. 2002.

[7] J. J. van Wijk, Seifertview, visualization of Seifert surfaces.

[8] A. Verweij, "Electrodynamics of superconducting cables in accelerator magnets," Ph.D. thesis, Univ. of Twente, Enschede, 1995.

[9] A. Jain, P. Wanderer, and R. Thomas et al., private communication, BNL, Brookhaven, NY, USA, Jun. 2006.

[10] J. Kaugerts, private communication, GSI, Darmstadt, Germany, Mar. 2005.

[11] L. Bottura, "A practical fit for the critical surface of Nb-Ti," IEEE Trans. Appl. Supercond., vol. 10, no. 1, pp. 1054-1057, Mar. 2000.

[12] N. Schwerg and C. Voellinger, "Development of a current fit function for $\mathrm{NbTi}$ to be used for calculation of persistent current induced field errors in the LHC main dipoles," IEEE Trans. Appl. Supercond., vol. 16, no. 2, pp. 1828-1831, Jun. 2006.

[13] B. Auchmann, "The coupling of discrete electromagnetism and the boundary element method for the simulation of accelerator magnets," $\mathrm{Ph} . \mathrm{D}$. thesis, Vienna Univ. of Technol., Vienna, 2004. 